\title{
Direct optical excitation of dark plasmons for hot electron generation
}

\author{
Niclas S. Mueller, (iD a Bruno G. M. Vieira, ${ }^{\text {ab }}$ Dominik Höing, ${ }^{c}$ \\ Florian Schulz, ${ }^{c}$ Eduardo B. Barros, ${ }^{c}$ Holger Lange (iD ${ }^{c}$ \\ and Stephanie Reich (iD *a
}

Received 9th October 2018, Accepted 12th November 2018

DOI: $10.1039 / c 8 f d 00149 a$

An ideal plasmonic system for hot-electron generation allows the optical excitation of plasmons, limits radiation losses, exhibits strong non-radiative electron damping, and is made from scalable and cost-effective materials. Here we demonstrate the optical excitation of dark interlayer plasmons in bilayers of colloidal gold nanoparticles. This excitation is created by an antiparallel orientation of the dipole moments in the nanoparticle layers; it is expected to exhibit strongly reduced radiative damping. Despite the vanishing dipole moment, an incoming electromagnetic wave that is propagating normal to the surface will excite the dark mode due to field retardation. We observe a strong peak in the absorption spectrum of a colloidal gold bilayer (nanoparticle diameter $=46 \mathrm{~nm}$ ); this peak is absent for a nanoparticle monolayer. The full width at half maximum of the dark mode is $230 \mathrm{meV}$ for an ideal nanoparticle crystal and 320 meV for the structure produced by self-assembly out of solution. The position and width of the dark plasmon are efficiently tailored by the interparticle distance within the layer, nanoparticle size and layer number. We present time-resolved pump and probe experiments of hot-electron generation by bright and dark bilayer nanoparticle modes.

\section{Introduction}

Plasmons are an excellent source of excited electrons. ${ }^{1-3}$ The excess energy of electrons may be used for driving catalytic reactions, in imaging applications and in photothermal heat generation, amongst other examples. ${ }^{2,4-9}$ Maximizing the yield of hot electrons requires careful engineering of the plasmonic decay channels so that dephasing is preferred over energy loss. In particular, relaxation channels that distribute a large amount of energy, such as photon emission or Auger-type processes, should be minimized.

\footnotetext{
${ }^{a}$ Department of Physics, Freie Universität Berlin, Arnimallee 14, 14195 Berlin, Germany. E-mail: sreich@zedat. fu-berlin.de

${ }^{b}$ Departamento de Física, Universidade Federal do Ceará, Fortaleza, Brazil

'Institute of Physical Chemistry, University of Hamburg, Martin-Luther-King-Platz 6, 20146 Hamburg, Germany
} 
Plasmon decay

$$
\Gamma=\Gamma_{\mathrm{b}}+\Gamma_{\mathrm{s}}+\Gamma_{\mathrm{r}}
$$

occurs via intrinsic (Drude) damping, $\Gamma_{\mathrm{b}}$, which includes all of the damping processes of the bulk metal, surface-mediated (Laundau) damping, $\Gamma_{\mathrm{s}}$, and radiative damping, $\Gamma_{\mathrm{r}}$, by photon scattering. ${ }^{\mathbf{1 , 1 0 - 1 3}}$ The desired relaxation channel for hot electron generation is $\Gamma_{\mathrm{s}}$, where the surface breaks the conservation of crystal momentum. ${ }^{\mathbf{1 1 4}}$ This mainly leads to dephasing with little energy loss, thus producing highly excited electrons.

One approach for optimising $\Gamma_{\mathrm{s}}$ is to reduce the size of the metal nanoparticles below the electron mean free path. ${ }^{1,15-18}$ Since light scattering by metal nanoparticles scales with the volume squared, a small diameter also reduces $\Gamma_{\mathrm{r}}$, making surface scattering a sizable contribution for particle diameters below $10 \mathrm{~nm} \cdot{ }^{\mathbf{1 1 , 1 2 , 1 5}}$ Bulk damping in gold is $\Gamma_{\mathrm{b}} \approx 100-200 \mathrm{meV}$ below $2.1 \mathrm{eV} ;{ }^{19} \Gamma_{\mathrm{s}}$ will add $20-30 \mathrm{meV}$ for an effective particle size of $10 \mathrm{~nm} \cdot{ }^{17,18}$ However, the small volumes also reduce the cross section for optical absorption. ${ }^{12}$ Since optical excitation is the most viable and economic route for plasmon excitations, a low absorption cross section reduces the total rate of hot electron generation per nanoparticle, even if the conversion of plasmons into hot electrons is highly efficient.

Another idea to increase plasmon relaxation via surface scattering is through the formation of hot spots in nanoparticle agglomerates and plasmonic oligomers. ${ }^{14,20-22}$ If two (or more) particles are in close contact, their plasmonic near fields interact, thus creating volumes of particularly high field enhancement (hot spots). ${ }^{12}$ Hot spots increase the rate of hot electron generation, because damping by surface scattering depends on the magnitude of the electric field close to the metal surface. ${ }^{1,21,23,24}$ A second effect is that the tight focusing of the field in a hot spot lifts the translational symmetry and thus the conservation of (quasi-) momentum. ${ }^{\mathbf{1 1 4}}$ The drawback of the second approach is the increase in radiative damping, $\Gamma_{\mathrm{r}}$. Optically active, "bright" plasmons decay rapidly by emitting photons into the far field. ${ }^{\mathbf{1 0 , 1 2}}$ Since the radiative decay channel is highly efficient for coupled nanoparticles, the plasmon lifetime is limited to a few fs and energy is lost to the radiative processes. ${ }^{25}$

Dark plasmons are plasmonic excitations with a vanishing net dipole moment, e.g., higher-order multipole modes in metal nanoparticles such as quadrupoles. $^{26,27}$ Assemblies of nanoparticles possess dark modes that arise by combining the dipolar plasmons of the individual particles into a collective mode with a vanishing dipole moment. ${ }^{28-30}$ Because dark plasmons have no net dipole moment, they typically do not interact with far field radiation; light is neither absorbed nor emitted/scattered. ${ }^{31}$ The absence of light emission is an advantage for hot-electron generation, because the entire excess energy is channelled into non-radiative processes, i.e. the creation of excited electrons. The absence of light absorption, on the other hand, is a major disadvantage because it prevents the optical excitation of dark modes. This disadvantage may be overcome, e.g. by exploiting Fano resonances for excitation in plasmonic oligomers, exciting the plasmon via a magnetic field instead of an electric field in metal-insulator-metal slabs, or exciting nanoparticle assemblies with cylindrical vector beams. ${ }^{30,32-34}$ These routes, however, require the nanofabrication of plasmonic nanostructures via electron-beam lithography and tailored beams of structured light. 
Here we demonstrate the excitation of dark modes and the creation of hot electrons using linearly polarized light and scalable, cost-effective plasmonic surfaces. The surfaces are created via the self-assembly of gold nanoparticles into colloidal mono- and bi-layers. Above a characteristic diameter $(30-50 \mathrm{~nm}$ depending on the surrounding medium), light absorption is altered by retardation, as we show through our symmetry analysis and numerical calculations. Light is absorbed by bright dipole modes and a dark mode in the bilayer that arises through the combination of the dipolar modes of the two layers into an excitation with a vanishing net dipole moment. We experimentally demonstrate dark plasmon absorption in gold bilayers. The hot-electron dynamics subsequent to plasmon excitation are analyzed via time-resolved pump-probe spectroscopy.

\section{Methods}

\section{Synthesis of colloidal gold nanoparticle layers}

The synthesis and characterization of gold mono- to few-layers is described in detail in ref. 35 and 36. To summarize, quasispherical citrate-stabilized gold nanoparticles with a diameter of $40 \mathrm{~nm}$ or $46 \mathrm{~nm}$ were synthesized based on the seeded-growth protocol. The particles were functionalized with thiolated polystyrene and mixed $(6 \mathrm{~mL})$ with polystyrene $(0.16 \mathrm{mM})$ and oleylamine $(1 \mathrm{mM})$ in toluene $(6 \mathrm{~mL})$ as well as ethanol $(6 \mathrm{~mL})$. After rapid stirring $(30 \mathrm{~min})$ and phase separation, the organic phase was extracted, concentrated and purified by centrifugation, resulting in a final gold nanoparticle concentration of $2.1 \mathrm{nM}$. The nanoparticle films were prepared via self-assembly at the liquid-liquid interface. For the transient absorption measurements, the nanoparticle films were deposited on quartz substrates. The gold nanoparticle solution in toluene was pipetted onto diethylene glycol in a Teflon well. The well was covered with a quartz slide and left undisturbed at room temperature until the solvent had evaporated and a gold nanoparticle film had formed..$^{35,36}$ For their characterization by transmission electron microscopy (TEM) and microabsorbance measurements, the films were carefully skimmed off with a carbon-coated copper grid. The probability of bilayer formation was increased by repeating the procedure. The grids were dried afterwards on filter paper for at least 24 hours.

\section{Microabsorbance spectroscopy}

Optical absorbance and reflectance spectra of the gold nanoparticle layers were recorded using a home-built microabsorbance spectrometer. For a detailed description of the optical setup, please see ref. 36. A supercontinuum laser (Fianium) was used as a broadband light source. The light was linearly polarized, the spectral range was reduced to the measurement range through a combination of long- and short-pass filters, and the total laser power was reduced to below 100 $\mu \mathrm{W}$. An inverted microscope was used to focus the light on the sample and find the region of interest (see Fig. 4c below). The light was focused on the substrate either by a $100 \times$ objective with a numerical aperture (NA) of 0.9 or by a $20 \times$ objective with an NA of 0.25 . The transmitted light was collected by objectives with a similar numerical aperture and detected by a fibre spectrometer (Avantes). As a reference for the transmittance, $T$, we recorded the transmission through the substrate without nanoparticle layers. The reflected light was separated from the 
incoming light by a beam splitter and guided into the spectrometer via an optical fibre. A silver mirror was used as a reference to obtain the reflectance, $R$. The absorbance spectrum was calculated as $1-T-R$. To determine the plasmon peak position and full width at half maximum (FWHM) we first subtracted the contribution from the gold interband transitions from the measured absorption spectrum. The absorption by the plasmons was fitted with two Lorentzian peaks (one peak for the monolayer that has only a bright mode).

\section{Transient absorption measurements}

For the transient absorption experiments, a commercial amplified Ti-sapphire laser system (Spitfire Ace, $800 \mathrm{~nm}, 1 \mathrm{kHz}, 35 \mathrm{fs}$; Spectra Physics) was employed as the main excitation source. Its output was split into two parts. One seeded an optical parametric amplifier (TOPAS-Prime; Light Conversion) that provides a tunable excitation wavelength over the range of 290-1600 $\mathrm{nm}$. The other output was used to generate the probe pulses. A commercial TA setup (Helios; Ultrafast Systems) was used to record the experiment. The quartz slides with the gold layers were mounted in a non-collinear geometry. The diameter of the focused probe beam was around $100 \mu \mathrm{m}$, whereas that of the pump beam was around $500 \mu \mathrm{m}$. Each differential spectrum was referenced by subtracting the ground-state spectrum from the pumped spectrum, using a $500 \mathrm{~Hz}$ chopper. The pump intensity was kept constant at $100 \mu \mathrm{J} \mathrm{cm}^{-2}(200 \mathrm{~nJ}$ per pulse).

\section{Finite-difference time-domain simulations}

Simulated spectra were obtained via the commercial software package, Lumerical FDTD Solutions. The layers were implemented by defining a unit cell and using periodic boundary conditions. Mesh-override regions of $0.25 \mathrm{~nm}$ were used for greater accuracy. The nanoparticle layers were illuminated by a plane-wave source. One power monitor was included behind the layers to capture the transmittance, $T$, and another was included behind the source to gather the reflectance, $R$. The absorbance, $A$, was later calculated as $A=1-T-R$. The gold dielectric functions were implemented by fitting the experimental data reported by Johnson and Christy. ${ }^{37}$ The nanoparticle layers were placed on top of a $10 \mathrm{~nm}$ thick amorphous carbon layer, as in the experiments, for the simulations shown in Fig. 5-7. The amorphous carbon was implemented by using the experimental data of Larruquert et al. ${ }^{38}$ A gold slab with a thickness of $200 \mathrm{~nm}$ was added for the simulation of the layers over a gold substrate, as shown in Fig. 9. Convergence tests were carried out by varying the mesh sizes in order to ensure the accuracy of the simulation results.

\section{Results and discussion}

\section{Optical selection rules for dark interlayer modes}

In order to understand the optical absorption by nanoparticle layers, we carried out a symmetry-based analysis of colloidal bilayers or crystals in a hexagonal close packed (hcp) structure. ${ }^{39,40}$ The hcp structure belongs to the $D_{6 \mathrm{~h}}$ point group; it has two nanoparticles in a hexagonal unit cell, one at the origin and the other at $(2 / 3,1 / 3,1 / 2)$, as shown in Fig. 1a. We will restrict our analysis to plasmon modes 

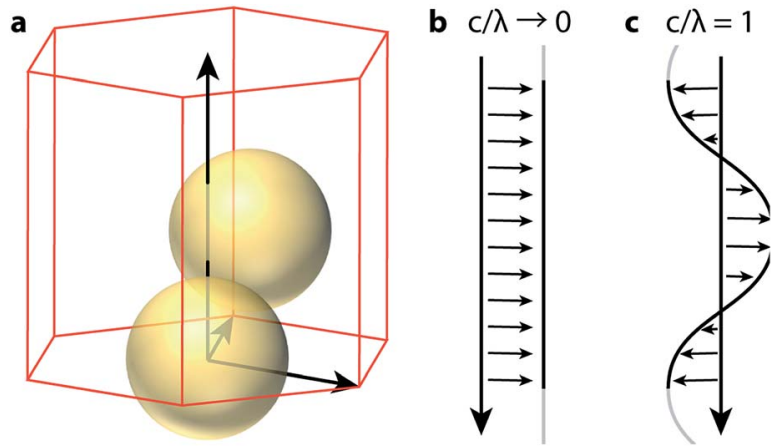

Fig. 1 (a) Unit cell of the hcp crystal with two nanoparticles in the unit cell. Electric field of the incoming light, assuming normal incidence under the assumption of (b) infinite wavelength and (c) a wavelength that matches the translational periodicity of the crystal shown in (a).

that arise as combinations of the dipole excitation in the nanoparticles; this argumentation extends straightforwardly to multipolar excitations.

The three-fold degenerate dipolar plasmons of the individual nanoparticles induce four dipole-derived eigenmodes in the bilayer ${ }^{39}$

$$
\Gamma_{\mathrm{pl}}^{\mathrm{dip}}=\mathrm{A}_{2 \mathrm{u}} \oplus \mathrm{B}_{2 \mathrm{~g}} \oplus \mathrm{E}_{1 \mathrm{u}} \oplus \mathrm{E}_{2 \mathrm{~g}}
$$

Two of these modes are non-degenerate, whilst two are two-fold degenerate. The symmetry-adapted eigenvectors are obtained by projection operators, which are shown in Fig. 2. Out of the representations detailed in eqn (2), the $A_{2 u}$ and $E_{1 u}$

\section{a bright eigenmodes, finite dipole moment}

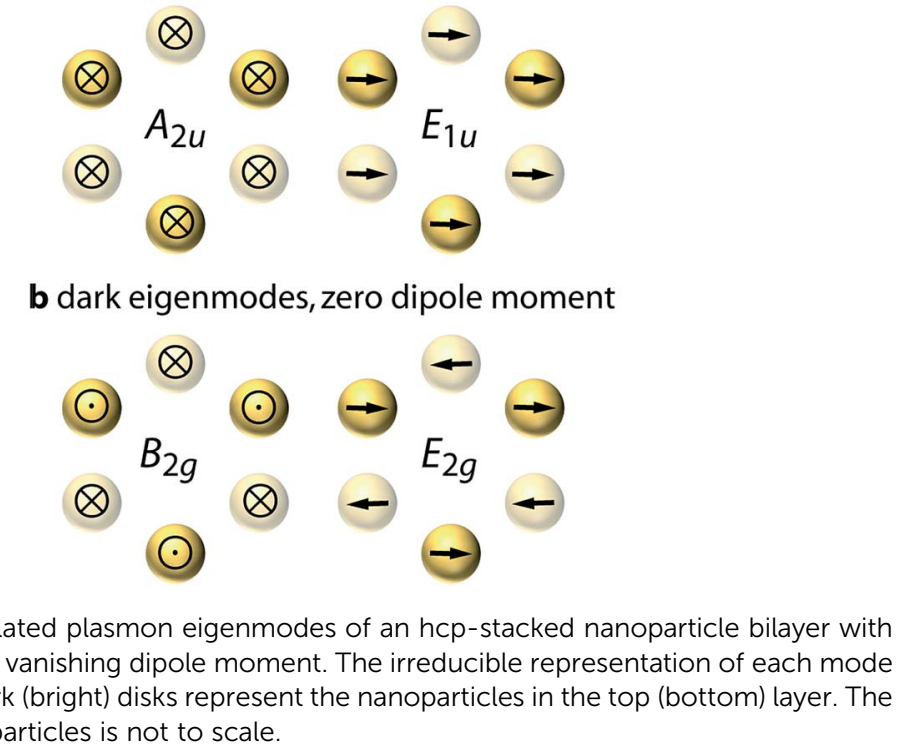

Fig. 2 Dipole-related plasmon eigenmodes of an hcp-stacked nanoparticle bilayer with a (a) finite and (b) vanishing dipole moment. The irreducible representation of each mode is shown. The dark (bright) disks represent the nanoparticles in the top (bottom) layer. The size of the nanoparticles is not to scale. 
representations are normally considered as optically active and have finite dipole moments, whereas $\mathrm{B}_{2 \mathrm{~g}}$ and $\mathrm{E}_{2 \mathrm{~g}}$ are considered dark (dipole forbidden). Within group theory, the selection rules for optical absorption are derived by analysing the transformation properties of the incoming electric field. ${ }^{39}$ The common approximations are to consider only the dipole expansion of the electric field and to assume the light wavelength to be large in comparison to the extension of the unit cell (Fig. 1b). Under this approximation, the electromagnetic field transforms like a vector, $\Gamma_{\mathrm{vec}}=\mathrm{A}_{2 \mathrm{u}} \oplus \mathrm{E}_{1 \mathrm{u}}$, thus leading to the selection rules listed above.

The assumption of infinite wavelength is justified for molecules and crystalline solids, because their characteristic lengths $(1 \mathrm{~nm})$ are much smaller than the wavelength of light (100-1000 nm). However, in the case of colloidal layers and crystals, the much larger extension of the unit cell $(10-100 \mathrm{~nm})$ makes retardation effects dominant. To examine the other limiting case, we consider the wavelength of the electromagnetic field to be identical to the translational symmetry of a colloidal crystal (Fig. 1c). For normal incidence, as shown in Fig. 1c, the field transforms according to $\mathrm{E}_{2 \mathrm{~g}}$. It will be able to excite the in-plane antiparallel dark mode shown on the right of Fig. $2 b$.

Symmetry analysis predicted the optical selection rules of the nanoparticle bilayers to depend on the ratio between the nanoparticle diameter, $d$, and the wavelength, $\lambda$, of the incoming light. For realistic nanoparticles, $d$ will always be much smaller than $\lambda$, but for at least some time during its period of oscillation, the electric field will be anti-parallel in the top and bottom layers. We expect this to activate absorption by the $\mathrm{E}_{2 \mathrm{~g}}$ dark mode.

To study the effect of retardation on the optical spectra, we simulated a gold nanoparticle bilayer in a vacuum with hcp stacking, a diameter, $d$, of $20 \mathrm{~nm}$, and

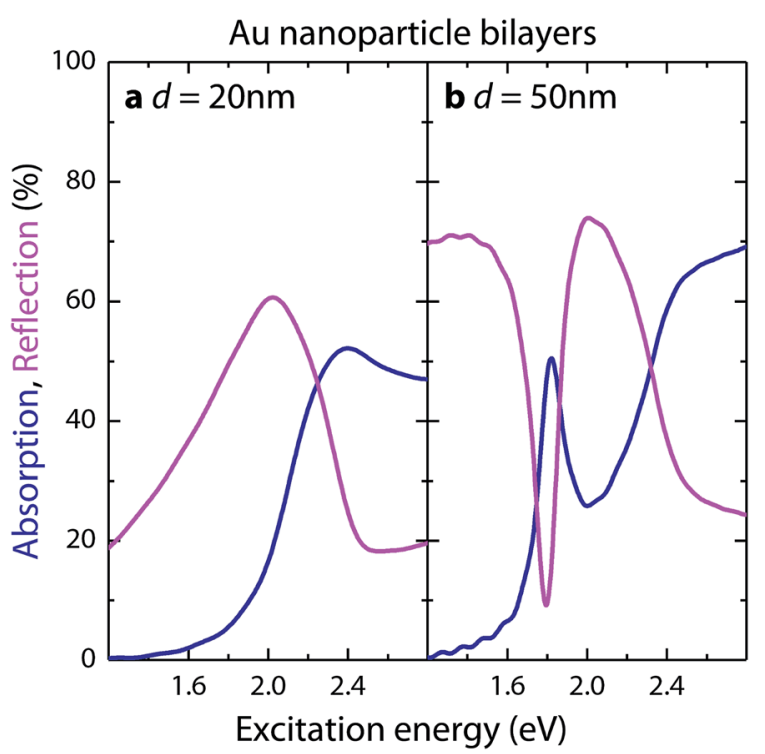

Fig. 3 Simulation of the absorption (blue) and reflection (magenta) of hcp-stacked gold nanoparticle bilayers with particle diameters of (a) $20 \mathrm{~nm}$ and (b) $50 \mathrm{~nm}$. The absorption peak at $1.82 \mathrm{eV}$ is caused by the dark plasmons. Gaps of $2 \mathrm{~nm}$ and a background medium with an index of $n=1$ were assumed. 
a gap size, $g$, of $2 \mathrm{~nm} .{ }^{41}$ The absorption spectrum (Fig. 3a) is dominated by the step function in the absorption coefficient due to interband transitions. ${ }^{42}$ The plasmon peak at $2.24 \mathrm{eV}$ overlaps with the interband transitions. It is strongly damped (FWHM $=\gamma=450 \mathrm{meV}$ ) and is hardly visible in the spectrum. Likewise, the reflection is broad and unstructured. After increasing the nanoparticle diameter to $50 \mathrm{~nm}$ (Fig. 3b), the simulation predicts a strong (50\%) absorption peak at $1.82 \mathrm{eV}$. It is accompanied by a drop in reflectance to almost zero, thus clearly identifying it as an electromagnetic resonance. We analysed the induced currents in the upper and lower layer in order to assign the plasmon eigenmode. ${ }^{36}$ Both currents point within the plane and are antiparallel. The peak at $1.82 \mathrm{eV}$ thus corresponds to the $\mathrm{E}_{2 \mathrm{~g}}$ eigenmode shown in Fig. $2 \mathrm{~b}$.

The FWHM of the $\mathrm{E}_{2 \mathrm{~g}}$ mode $\left(\gamma\left(\mathrm{E}_{2 \mathrm{~g}}\right)=140 \mathrm{meV}\right)$ is much narrower than the width of the bright $\mathrm{E}_{1 \mathrm{u}}$ mode $\left(\gamma\left(\mathrm{E}_{1 \mathrm{u}}\right)=710 \mathrm{meV}\right)$ which is observed at $E\left(\mathrm{E}_{1 \mathrm{u}}\right)=$ $2.07 \mathrm{eV}$ for the $50 \mathrm{~nm}$ spheres. The bulk damping, $\Gamma_{\mathrm{b}}$, of the gold nanoparticles is $110 \mathrm{meV}$ at $E\left(\mathrm{E}_{2 \mathrm{~g}}\right)=1.82 \mathrm{eV}$ and $200 \mathrm{meV}$ at $E\left(\mathrm{E}_{1 \mathrm{u}}\right)=2.07 \mathrm{eV} \cdot{ }^{19}$ This implies an additional damping of only $30 \mathrm{meV}$ for the dark $\mathrm{E}_{2 \mathrm{~g}}$ plasmon mode, but $510 \mathrm{meV}$ for the bright $\mathrm{E}_{1 \mathrm{u}}$ mode. The additional broadening is caused by radiative damping where a large fraction of the absorbed energy is lost in the form of photons. The dark modes of the colloidal bilayers are potential excitations for minimizing radiation loss and maximizing the decay of plasmons into hot carriers for harvesting losses.

\section{Absorption by dark interlayer plasmons}

The excitation of dark modes through field retardation was predicted for nanoparticle diameters of $\gtrsim 50 \mathrm{~nm}$. We recently developed an advanced synthesis technique for $d=46 \mathrm{~nm}$ gold particles and their assembly into colloidal hcp structures (see the methods section in ref. 35). Transmission electron microscopy (TEM) images show hexagonal packing due to self-assembly after drying the solution on a TEM grid with a $10 \mathrm{~nm}$ amorphous carbon film (Fig. 4a). ${ }^{36}$ Parts of the sample were areas with bi-, tri- and few-layers (Fig. 4b) that often extended
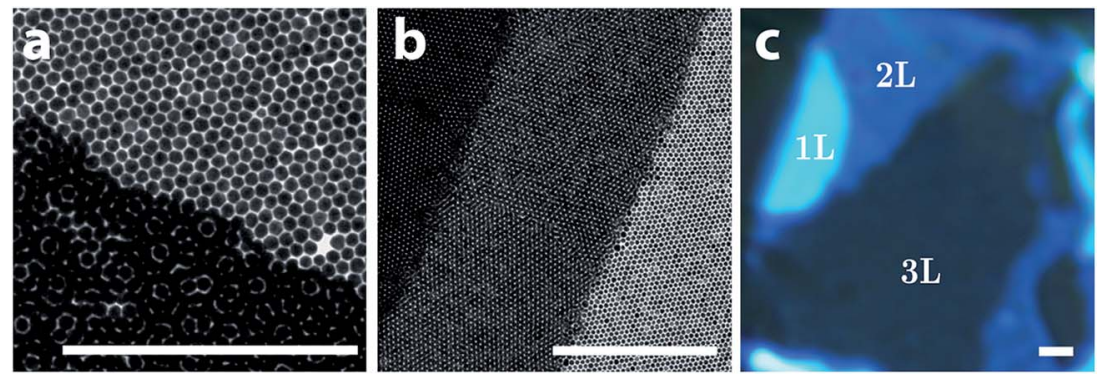

Fig. 4 (a) TEM image of a colloidal gold monolayer (top) and bilayer (bottom). The monolayer demonstrates a well-ordered structure with little defects (missing particles and smaller particles). The Moire pattern in the bilayer indicates a finite angle of rotation between the two layers. (b) Survey TEM image of a monolayer (right), bilayer (middle) and trilayer (left). (c) The number of layers may also be determined via analysis of the change in contrast in the optical microscopy images, as shown for a sample spot with a monolayer (1L), bilayer (2L) and trilayer (3L). The scale bars correspond to $1 \mu \mathrm{m}$. 
over many $\mu \mathrm{m}^{2}$ (Fig. $4 \mathrm{c}$ ). ${ }^{35} \mathrm{~A}$ change in the optical contrast with changing layer number was also clearly visible via optical microscopy measurements (Fig. 4c). It allows the identification of the number of layers during the absorption and reflection measurements.

Fig. 5 shows the measured and simulated absorption spectra of a gold nanoparticle monolayer. It features a weak plasmon peak in the background of the interband transitions similar to the bilayer calculations of the $20 \mathrm{~nm}$ particles presented above. For the bilayers (Fig. 6), the absorption spectra are dominated by the $\mathrm{E}_{2 \mathrm{~g}}$ peak at $E\left(\mathrm{E}_{2 \mathrm{~g}}\right)=1.52 \mathrm{eV}$. The peak is at a lower frequency than in Fig. 3, because the nanoparticles are stabilized by polystyrene and oleylamine molecules, thus giving rise to a background refractive index of $n=1.4$. We recorded the spectra of various bilayers and consistently obtained almost identical spectra (see Fig. $6 \mathrm{~b}$ and $\mathrm{c}$ for two spots measured with a $100 \times$ objective). This shows that the system is insensitive towards crystal imperfections such as missing nanoparticles, grain boundaries in the hexagonal packing, and the stacking of the two layers. ${ }^{36}$ This robust behaviour is understandable when considering the macroscopic nature of the plasmon mode, whose main feature is the antiparallel dipole orientation in the two layers. The excitation of dark plasmons due to retardation is not limited to bilayers, but can be expected for any multilayer arrangement of plasmonic nanoparticles. In general, a sample with $m$ gold nanoparticle layers will have $(m-1)$ absorption peaks by dark plasmons in the visible and near infrared when excited with linearly polarized light. ${ }^{36}$

We evaluated the FWHM of the $\mathrm{E}_{1 \mathrm{u}}$ and $\mathrm{E}_{2 \mathrm{~g}}$ plasmons for the gold monolayer and bilayer (see Table 1). The energetic position of the plasmon shows excellent

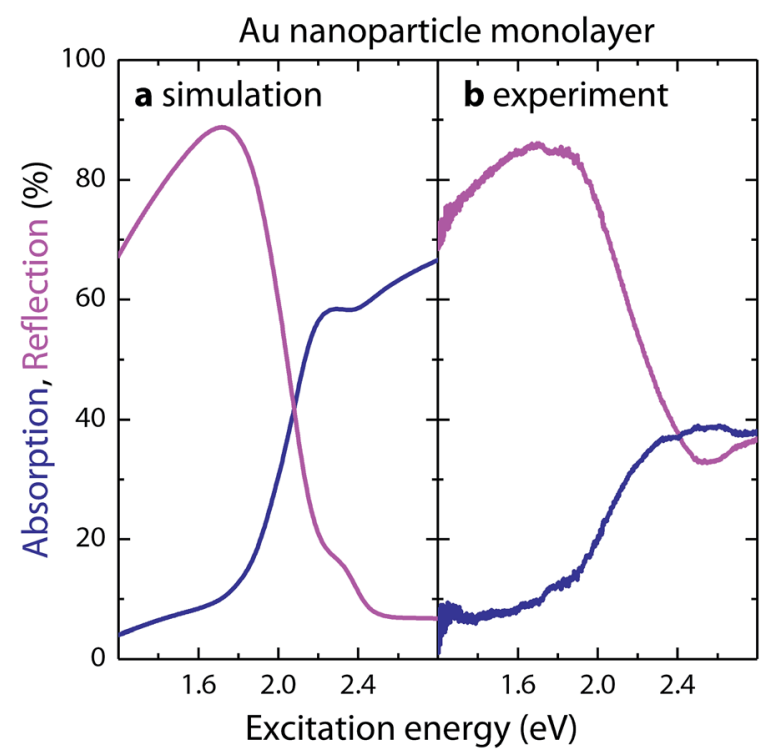

Fig. 5 (a) Simulated and (b) measured absorption (blue) and reflection (magenta) spectra of a gold monolayer with $d=46 \mathrm{~nm}$ and $g=2 \mathrm{~nm}$. The simulation used a background dielectric constant with $n=1.4$ and assumed hcp stacking. The nanoparticle layer was placed on top of a $10 \mathrm{~nm}$ thick amorphous carbon film. 
Au nanoparticle bilayer

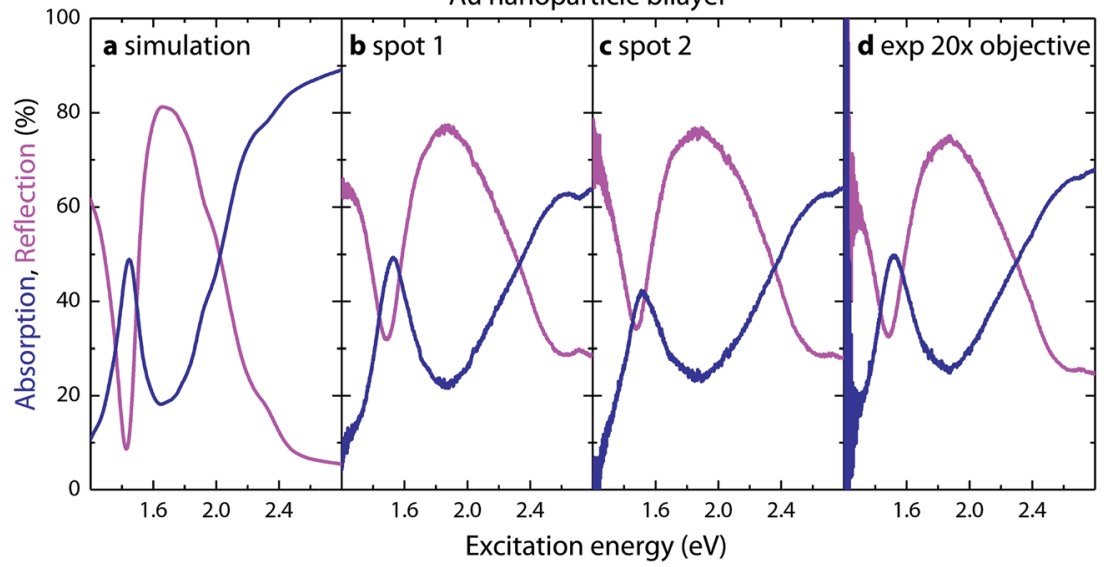

Fig. 6 Absorption (blue) and reflection (magenta) spectra of gold nanoparticle bilayers. (a) Simulation, (b and c) two spots measured with a $100 \times$ objective, and (d) a spot measured with a $20 \times$ objective. The peaks at $1.52 \mathrm{eV}$ are caused by the dark $\mathrm{E}_{2 \mathrm{~g}}$ plasmons. The parameters are identical to those reported for Fig. 5.

agreement between the experimental and simulation results for the gold monolayer. For the bilayer, the position of the dark mode was underestimated and the position of the bright mode overestimated in the simulations. The widths of the plasmon peaks for the monolayer and bilayer are consistently $100 \mathrm{meV}$ broader in the experimental results than those predicted by the simulations. The contributions to this additional width arise from surface scattering and inhomogeneous broadening due to size and shape variations. ${ }^{17,18,35}$

Variations in the particle diameter (estimated as $46 \pm 3 \mathrm{~nm}$ from analysis of the TEM images) lead to variations in the interparticle gap widths ( $g=2 \pm 1 \mathrm{~nm}$ ). The distance between the nanoparticles strongly affects the position of the $\mathrm{E}_{2 \mathrm{~g}}$ mode, thus explaining the larger linewidth. ${ }^{41}$ We find that the spectra measured with a $100 \times$ and $20 \times$ objective exhibit identical peak positions and FWHM values (Table 1 and Fig. 6b-d). ${ }^{36}$ Inhomogeneous broadening must therefore happen at a length of well below $1 \mu \mathrm{m}$. On the other hand, the position of the bright mode is hardly affected by variation in the particle size and interparticle distance. ${ }^{41}$ The additional broadening of both modes, therefore, rather points at least partially to an intrinsic mechanism such as surface scattering enhanced by hot spot

Table 1 Energy, $E$, and damping, $\gamma$, of the dark $\left(E_{2 g}\right)$ and bright $\left(E_{1 u}\right)$ modes of the monolayer and bilayer

\begin{tabular}{lllll}
\hline & $E\left(\mathrm{E}_{2 \mathrm{~g}}\right)$ in eV & $\gamma\left(\mathrm{E}_{2 \mathrm{~g}}\right)$ in $\mathrm{meV}$ & $E\left(\mathrm{E}_{1 \mathrm{u}}\right)$ in eV & $\gamma\left(\mathrm{E}_{1 \mathrm{u}}\right)$ in $\mathrm{meV}$ \\
\hline Monolayer, 100× & - & - & $2.18 \pm 0.05$ & $510 \pm 50$ \\
Monolayer, & - & - & 2.16 & 400 \\
simulation & & & & \\
Bilayer, 100 & $1.524 \pm 0.005$ & $325 \pm 15$ & $2.04 \pm 0.03$ & $630 \pm 20$ \\
Bilayer, 20× & $1.52 \pm 0.01$ & $330 \pm 30$ & $2.07 \pm 0.05$ & $620 \pm 50$ \\
Bilayer, simulation & 1.44 & 230 & 2.14 & 510
\end{tabular}


formation. ${ }^{\mathbf{1} 14}$ Measurements of the near-field resonance via scanning optical microscopy as a function of wavelength will help in clarifying this point.

\section{Time-resolved transient absorption spectroscopy}

To measure the electron dynamics in the gold nanoparticle layers, we utilized time-resolved transient absorption (TTA) spectroscopy. In these experiments, a probe pulse monitors the transient change in absorption in the visible (around the $\mathrm{E}_{1 \mathrm{u}}$ energy) region in order to follow the temperature increase in the electronic system after the absorption of a strong pump pulse. Fig. 7a displays exemplary TTA spectra with excitation at $1.27 \mathrm{eV}$, i.e. away from any pronounced resonance of the gold layers. The spectra are dominated by the response of the bilayer $(d=40$ $\mathrm{nm}$ ). The sample consisted of bi- and penta-layer domains, but no visible light was transmitted through the pentalayers over the spectral range of the probe pulse. We observe a temperature increase of the electron gas after plasmon excitation and dephasing; this results in a broadening of the $\mathrm{E}_{1 \mathrm{u}}$ absorption, ${ }^{43}$ which generates a pronounced bleach with two positive sidebands in the differential spectrum $^{44}$ (Fig. 7a). The coupled plasmon modes at the long-wavelength tail behave differently, thus resulting in asymmetry of the positive sidebands. The intensity of the main bleach can be related to the electron temperature, but it is sensitive to the experimental conditions.

The dynamics of the main bleach can be separated into three phases with different timescales: contrast increase after plasmon dephasing and electronelectron scattering (10 fs), electron-phonon coupling (1 ps), and cooling of the nanoparticle $(10 \mathrm{ps})^{45}$ (see Fig. $\left.7 \mathrm{~b}\right)$. The data were evaluated with a bi-exponential fit to obtain the characteristic time constants. Due to the linear heat capacity and constant electron-phonon coupling, the observed electron-phonon coupling time constant, $t_{1}$, can be directly related to the initial excess temperature in the electron gas..$^{434}$ This quantity is less sensitive to experimental variations than the bleach intensity.
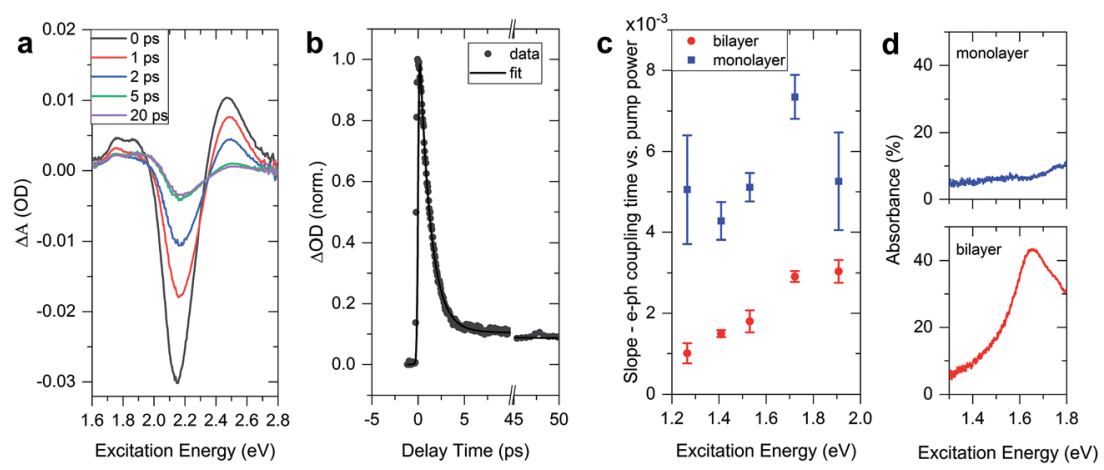

Fig. 7 Time-resolved transient absorption (TTA) spectroscopy of gold nanoparticle layers $(d=40 \mathrm{~nm}, g=2 \mathrm{~nm})$. (a) TTA spectra with excitation at $1.27 \mathrm{eV}$ for selected pump-probe delays. (b) Normalized intensity of the main bleach shown in (a). (c) Slope of the electronphonon coupling constant, $t_{1}$, versus pump power for different pump energies measured for a monolayer (blue) and a bi- and few-layer (red). (d) Absorbance spectra of the monolayer (top, blue) and the bi- and few-layer (bottom, red). 
As the initial temperature scales linearly with the photon flux, the slope of the electron-phonon coupling time (initial temperature) with photon input allows us to assess the efficiency of the hot electron generation (Fig. 7c). We carried out a general screening of the excitation-dependence between 1.2 and $2.0 \mathrm{eV}$. The monolayer shows no dependence on the excitation energy, which is in agreement with its structure-less absorption spectrum (Fig. 7d). In the bilayer we observe an increase in the hot-electron generation efficiency at $1.65 \mathrm{eV}$, which corresponds to the $\mathrm{E}_{2 \mathrm{~g}}$ resonance for this bilayer with $d=40 \mathrm{~nm}$, as shown in Fig. 7d. The exact dependence and origin of the increase of the slope of the electron-phonon coupling constant is the subject of ongoing studies.

\section{Optimization of nanostructure design for hot electron generation}

Finally, we will show that the nanostructure design can be further optimized to increase the hot electron yield by dark interlayer plasmon excitation. The surface mediated damping, $\Gamma_{\mathrm{s}}$, which is relevant for hot-electron generation, increases with decreasing nanoparticle size..$^{\mathbf{1 4 , 1 7 , 1 8}}$ The quantum yield of hot electrons in single metal nanoparticles decreases with increasing particle size, but remains relatively large for diameters up to $30 \mathrm{~nm}$. In Fig. 8a we show by simulation how the absorbance spectra of gold nanoparticle bilayers evolve with decreasing particle diameter. The narrow $\mathrm{E}_{2 \mathrm{~g}}$ absorbance peak remains well resolved down to $d=20 \mathrm{~nm}$. This is because the background refractive index $(n=1.4)$ reduces the incident wavelength and increases field retardation. With decreasing particle diameter, radiative damping is further suppressed thus reducing the width of the dark mode. The dark mode can be characterized by the quality factor $Q=E\left(\mathrm{E}_{2 \mathrm{~g}}\right) /$ $\gamma\left(E_{2 g}\right)$, which is associated with the field enhancement. ${ }^{10,19}$ The highest quality factor $(Q=22.5)$ is expected for a nanoparticle bilayer with $d=25 \mathrm{~nm}$ (see the disks in Fig. 8b). This is much larger than the quality factor $(Q<8)$ of isolated spherical gold nanoparticles of similar size ${ }^{\mathbf{1 0}}$ in fact, $Q$ reaches its limit for gold nanostructures that is imposed by $\Gamma_{\mathrm{b}}{ }^{19}$ For $d=10 \mathrm{~nm}$, the width of the
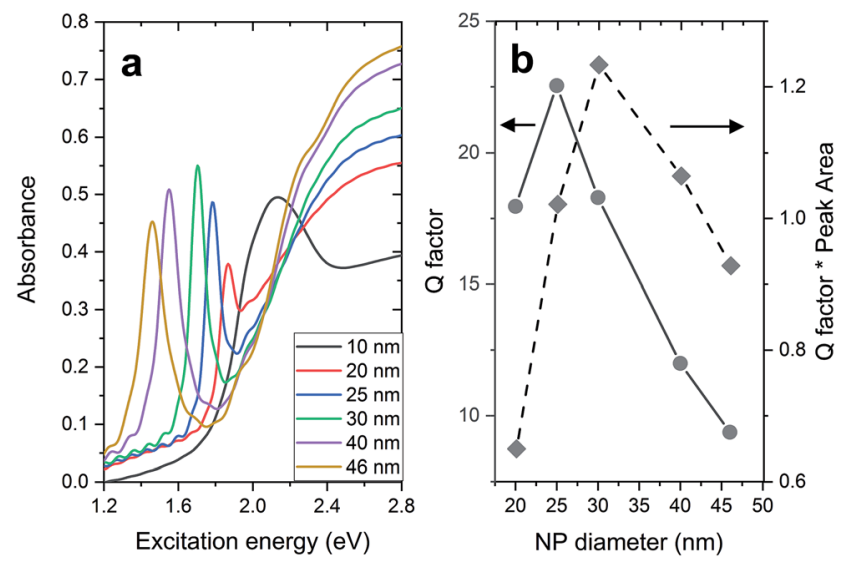

Fig. 8 Excitation of dark interlayer plasmons in gold nanoparticle bilayers with different particle sizes. (a) Simulated optical absorbance of gold nanoparticle bilayers with different particle diameters. Interparticle gaps of $2 \mathrm{~nm}$ and a background index of $n=1.4$ were assumed. (b) Quality factor, $Q=E\left(E_{2 g}\right) / \gamma\left(E_{2 g}\right)$, and its product with the peak area, $A\left(E_{2 g}\right)$, as a function of nanoparticle diameter. 


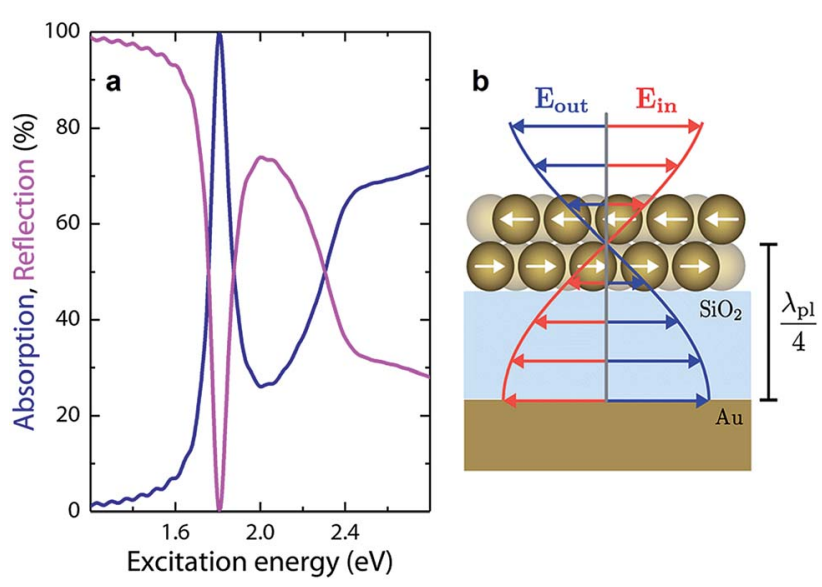

Fig. 9 Increasing the dark mode absorption to $100 \%$ by destructive interference above a gold mirror. (a) Simulated absorbance (blue) and reflectance (magenta) spectra of a $46 \mathrm{~nm}$ gold nanoparticle bilayer placed on top of a gold substrate with a $102 \mathrm{~nm}$ thick $\mathrm{SiO}_{2}$ layer. Interparticle gaps of $2 \mathrm{~nm}$ and a background index of $n=1.4$ were assumed. (b) Explanation of the increase in absorbance. The transmitted and reflected light is cancelled out by destructive interference when the optical distance between the bilayer center and gold surface is a quarter of the dark plasmon wavelength.

absorption peak strongly increases. This is partially due to the strong damping by the onset of the interband transitions, but an in-depth analysis of the simulations is necessary to understand this behaviour.

The second important parameter for the generation rate of hot electrons is the cross section for optical absorption. Considering both the quality factor and absorption (see the diamonds in Fig. 8b), we identify $30 \mathrm{~nm}$ as the most promising nanoparticle diameter for the generation of hot electrons in colloidal bilayers. Measuring the steady-state and time-resolved absorption of bilayers with varying nanoparticle diameter will be a way to verify our prediction.

Finally, we demonstrate that the optical absorption cross section of the dark plasmons can be significantly increased when placed on top of a reflective surface. ${ }^{46,47}$ We simulate the optical absorbance of a gold nanoparticle bilayer on top of a gold substrate with a $102 \mathrm{~nm}$ thick $\mathrm{SiO}_{2}$ layer. For a $\mathrm{SiO}_{2}$ thickness that puts the inversion center of the bilayer at $\lambda_{\mathrm{pl}} / 4$ over the gold surface $\left(\lambda_{\mathrm{pl}}\right.$ is the plasmon wavelength), the absorption reaches $100 \%$ (Fig. 9a). This striking increase in comparison with Fig. 6a is explained by a consideration of the destructive optical interference of the transmitted and reflected light, as illustrated in Fig. 9b. The colloidal gold surface may be optimized for perfect absorption by nanoparticles with different diameters by adjusting the thickness of the $\mathrm{SiO}_{2}$ layer to the dark plasmon wavelength. The perfect optical absorption in combination with the suppressed radiative damping will enable the efficient generation of hot electrons for photocatalysis and plasmon-mediated chemical reactions.

\section{Conclusions}

In conclusion, we studied the optical excitation and decay of dark modes as a source of hot electrons. Dark plasmons may be excited in colloidal gold bilayers through the 
use of linearly polarized light under normal incidence. This activation of optical absorption is explained by field retardation and the comparatively large size of the colloidal gold nanoparticles. We showed that the damping of dark modes is strongly reduced in comparison to that of bright plasmons, because radiative damping is absent. The generation of plasmonic hotspots in hexagonal close packed crystals instead favours the decay by surface effects exciting hot electrons. We discussed how to maximize the rate of hot electron emission by increasing the absorption efficiency of the dark plasmons to $100 \%$ and reducing the nanoparticle size to make surface effects dominant. Colloidal multilayers can be prepared over larger areas and hold potential as large-scale and low-cost substrates for hot-electron harvesting.

\section{Conflicts of interest}

There are no conflicts of interest to declare.

\section{Acknowledgements}

This work was supported by the European Research Council under grant DarkSERS (772108). N. S. M. acknowledges Deutsche Telekom Stiftung for their financial support. B. G. M. V. acknowledges the Coordenação de Aperfeiçoamento de Pessoal de Nivel Superior (CAPES) for their financial support under the program PDSE (Grant No. 88881.134611/2016-01) and Dahlem Research School (DRS). E. B. B. acknowledges the financial support from CNPq. F. S. was supported by the DFG via the project SCHU 3019/2-1. H. L. acknowledges the German Research Foundation (DFG) for their financial support through the Cluster of Excellence CUI.

\section{References}

1 G. V. Hartland, L. V. Besteiro, P. Johns and A. O. Govorov, ACS Energy Lett., 2017, 2, 1641-1653.

2 M. L. Brongersma, N. J. Halas and P. Nordlander, Nat. Nanotechnol., 2015, 10, 25.

3 S. Lal, S. E. Clare and N. J. Halas, Acc. Chem. Res., 2008, 41, 1842-1851.

4 S. Linic, P. Christopher and D. B. Ingram, Nat. Mater., 2011, 10, 911.

5 C. Clavero, Nat. Photonics, 2014, 8, 95.

6 S. Linic, U. Aslam, C. Boerigter and M. Morabito, Nat. Mater., 2015, 14, 567.

7 X. Huang, I. H. El-Sayed, W. Qian and M. A. El-Sayed, J. Am. Chem. Soc., 2006, 128, 2115-2120.

8 A. M. Schwartzberg and J. Z. Zhang, J. Phys. Chem. C, 2008, 112, 10323-10337.

9 P. Narang, R. Sundararaman and A. Atwater Harry, Nanophotonics, 2016, 596.

10 C. Sönnichsen, T. Franzl, T. Wilk, G. von Plessen, J. Feldmann, O. Wilson and P. Mulvaney, Phys. Rev. Lett., 2002, 88, 077402.

11 A. Crut, P. Maioli, N. Del Fatti and F. Vallée, Chem. Soc. Rev., 2014, 43, 39213956.

12 S. A. Maier, Plasmonics: Fundamentals and Applications, Springer US, 2010.

13 M. Liu, M. Pelton and P. Guyot-Sionnest, Phys. Rev. B: Condens. Matter Mater. Phys., 2009, 79, 035418.

14 L. V. Besteiro and A. O. Govorov, J. Phys. Chem. C, 2016, 120, 19329-19339.

15 J. A. Scholl, A. L. Koh and J. A. Dionne, Nature, 2012, 483, 421. 
16 S. Link and M. A. El-Sayed, J. Phys. Chem. B, 1999, 103, 8410-8426.

17 C. Novo, D. Gomez, J. Perez-Juste, Z. Zhang, H. Petrova, M. Reismann, P. Mulvaney and G. V. Hartland, Phys. Chem. Chem. Phys., 2006, 8, 35403546.

18 S. Berciaud, L. Cognet, P. Tamarat and B. Lounis, Nano Lett., 2005, 5, 515-518.

19 F. Wang and Y. R. Shen, Phys. Rev. Lett., 2006, 97, 206806.

20 P. Christopher, H. Xin, A. Marimuthu and S. Linic, Nat. Mater., 2012, 11, 1044.

21 A. Sousa-Castillo, M. Comesaña-Hermo, B. Rodríguez-González, M. PérezLorenzo, Z. Wang, X.-T. Kong, A. O. Govorov and M. A. Correa-Duarte, J. Phys. Chem. C, 2016, 120, 11690-11699.

22 H. Harutyunyan, A. B. F. Martinson, D. Rosenmann, L. K. Khorashad, L. V. Besteiro, A. O. Govorov and G. P. Wiederrecht, Nat. Nanotechnol., 2015, 10, 770 .

23 H. Zhang and A. O. Govorov, J. Phys. Chem. C, 2014, 118, 7606-7614.

24 X.-T. Kong, Z. Wang and A. O. Govorov, Adv. Opt. Mater., 2016, 5, 1600594.

25 O. L. Muskens, V. Giannini, J. A. Sánchez-Gil and J. Gómez Rivas, Opt. Express, 2007, 15, 17736-17746.

26 C. F. Bohren and D. R. Huffman, Absorption and scattering of light by small particles, Wiley, 1983.

27 M. Liu, T.-W. Lee, S. K. Gray, P. Guyot-Sionnest and M. Pelton, Phys. Rev. Lett., 2009, 102, 107401.

28 P. Nordlander, C. Oubre, E. Prodan, K. Li and M. I. Stockman, Nano Lett., 2004, 4, 899-903.

29 J. A. Fan, C. Wu, K. Bao, J. Bao, R. Bardhan, N. J. Halas, V. N. Manoharan, P. Nordlander, G. Shvets and F. Capasso, Science, 2010, 328, 1135.

30 J. Sancho-Parramon and S. Bosch, ACS Nano, 2012, 6, 8415-8423.

31 M.-W. Chu, V. Myroshnychenko, C. H. Chen, J.-P. Deng, C.-Y. Mou and F. J. García de Abajo, Nano Lett., 2009, 9, 399-404.

32 B. Luk'yanchuk, N. I. Zheludev, S. A. Maier, N. J. Halas, P. Nordlander, H. Giessen and C. T. Chong, Nat. Mater., 2010, 9, 707.

33 T. Pakizeh, M. S. Abrishamian, N. Granpayeh, A. Dmitriev and M. Käll, Opt. Express, 2006, 14, 8240-8246.

34 R. Verre, Z. J. Yang, T. Shegai and M. Käll, Nano Lett., 2015, 15, 1952-1958.

35 F. Schulz, S. Tober and H. Lange, Langmuir, 2017, 33, 14437-14444.

36 N. S. Mueller, B. G. d. M. Vieira, F. Schulz, P. Kusch, V. Oddone, E. B. Barros, H. Lange and S. Reich, ACS Photonics, 2018, 5, 3962.

37 P. B. Johnson and R. W. Christy, Phys. Rev. B: Condens. Matter Mater. Phys., 1972, 6, 4370-4379.

38 J. I. Larruquert, L. V. Rodríguez-de Marcos, J. A. Méndez, P. J. Martin and A. Bendavid, Opt. Express, 2013, 21, 27537-27549.

39 T. Inui, Y. Tanabe and Y. Onodera, Group theory and its applications in physics, Springer-Verlag, 1990.

40 D. W. Brandl, N. A. Mirin and P. Nordlander, J. Phys. Chem. B, 2006, 110, 12302-12310.

41 B. G. d. M. Vieira, N. S. Mueller, E. B. Barros and S. Reich, 2018, in preparation.

42 P. Anatoliy, P. Gero von and K. Uwe, J. Phys. D: Appl. Phys., 2004, 37, 3133.

43 G. V. Hartland, Chem. Rev., 2011, 111, 3858-3887.

44 A. M. Brown, R. Sundararaman, P. Narang, W. A. Goddard and H. A. Atwater, Phys. Rev. B, 2016, 94, 075120. 
45 E. Minutella, F. Schulz and H. Lange, J. Phys. Chem. Lett., 2017, 8, 4925-4929. 46 A. Moreau, C. Ciraci, J. J. Mock, R. T. Hill, Q. Wang, B. J. Wiley, A. Chilkoti and D. R. Smith, Nature, 2012, 492, 86.

47 C. Ng, J. J. Cadusch, S. Dligatch, A. Roberts, T. J. Davis, P. Mulvaney and D. E. Gomez, ACS Nano, 2016, 10, 4704-4711. 